\title{
Long-term results of oral valganciclovir for treatment of anterior segment inflammation secondary to cytomegalovirus infection
}

This article was published in the following Dove Press journal:

Clinical Ophthalmology

16 April 2012

Number of times this article has been viewed

\section{Victoria WY Wong \\ Carmen KM Chan \\ Dexter YL Leung \\ Timothy YY Lai}

Department of Ophthalmology and Visual Sciences, The Chinese University of Hong Kong, Hong Kong Eye Hospital, Hong Kong, People's Republic of China
Correspondence: Timothy YY Lai Department of Ophthalmology and Visual Sciences, The Chinese University of Hong Kong, Hong Kong Eye Hospital, I47K Argyle Street, Kowloon, Hong Kong SAR, China

Tel +852 27623134

Fax +852 27I5 9490

Email tyylai@cuhk.edu.hk
Background: The purpose of this study was to assess the efficacy of oral valganciclovir in the treatment of anterior segment inflammation caused by cytomegalovirus (CMV) infection.

Methods: Consecutive patients with anterior segment inflammation due to CMV causing anterior uveitis or corneal endotheliitis treated with oral valganciclovir were reviewed. Diagnosis of CMV infection was confirmed by polymerase chain reaction of the aqueous aspirate prior to commencement of oral valganciclovir. All patients were treated with an oral loading dose of $900 \mathrm{mg}$ valganciclovir twice daily for at least 2 weeks, followed by an additional $450 \mathrm{mg}$ valganciclovir twice-daily maintenance therapy. Changes in visual acuity, intraocular pressure (IOP), use of antiglaucomatous eye drops, and recurrence were analyzed.

Results: Thirteen eyes of 11 patients were followed for a mean of 17.2 months. Two patients had bilateral corneal endotheliitis. All eyes had absence of anterior segment inflammation within 3 weeks after treatment. Following treatment, the mean $\log M A R$ visual acuity improved significantly from 0.58 at baseline to 0.37 at the last follow-up $(P=0.048)$. The mean IOP and number of antiglaucomatous eye drops also decreased significantly $(P=0.021$ and $P=0.004$, respectively). Five $(38.5 \%)$ eyes had recurrence of anterior uveitis after valganciclovir was stopped and required retreatment with oral valganciclovir.

Conclusion: Oral valganciclovir appeared to be effective in controlling CMV anterior uveitis, resulting in visual improvement and IOP reduction following control of inflammation. However, despite the initial clinical response in all cases, recurrence after cessation of oral valganciclovir could occur.

Keywords: cytomegalovirus infection, inflammation, anterior uveitis, endotheliitis, ocular hypertension, antiviral, valganciclovir

\section{Introduction}

In recent years, with the increasing use of polymerase chain reaction (PCR) for the detection of viruses in intraocular fluids, cytomegalovirus (CMV) infection is increasingly recognized as an important cause of anterior segment inflammation in immunocompetent individuals. ${ }^{1-5}$ Clinical features of CMV anterior segment inflammation in immunocompetent individuals include increased intraocular pressure (IOP), with anterior chamber inflammation like Posner-Schlossmann syndrome, iris atrophy with nodular endothelial changes similar to Fuchs uveitis syndrome, and corneal endotheliitis with coin-shaped keratic precipitates with or without corneal edema. ${ }^{6-10}$ Systemic and ocular antiviral therapies with ganciclovir are the main treatment options for CMV anterior segment inflammation., ${ }^{4,5,10-12}$ Valganciclovir, the prodrug of ganciclovir, is also available for convenient oral dosing instead of 
intravenous ganciclovir. ${ }^{13,14}$ Most previous studies have only evaluated the short-term outcome of oral valganciclovir in the treatment of CMV anterior segment inflammation. The purpose of our study was to evaluate the long-term efficacy of oral valganciclovir in the treatment of CMV anterior segment inflammation.

\section{Materials and methods}

The study was a retrospective case series carried out at the Hong Kong Eye Hospital, Hong Kong. The case notes of all consecutive immunocompetent patients with CMV anterior segment inflammation who received oral valganciclovir treatment between June 2007 and August 2010 with a minimum follow-up duration of 6 months were reviewed. CMV anterior segment inflammation was defined as PCRconfirmed CMV infection together with clinical features of Posner-Schlossmann syndrome, Fuchs uveitis syndrome, or corneal endotheliitis. Exclusion criteria included patients with human immunodeficiency virus infection, and those on systemic chemotherapy or immunosuppressants for internal organ transplantation or connective tissue disease. All patients received a loading dose of oral valganciclovir $900 \mathrm{mg}$ twice daily for at least two weeks, followed by maintenance therapy of valganciclovir $450 \mathrm{mg}$ twice daily for at least 6 weeks. The study was performed in accordance with the tenets of the Declaration of Helsinki.

The main outcome measures of the study included baseline and final visual acuity, IOP measurements, use of antiglaucomatous drugs, and details of anterior segment inflammation on clinical examination, such as anterior chamber cells, presence of keratic precipitates, and iris atrophy.

\section{Statistical analysis}

Demographic characteristics of the patients are summarized by descriptive statistics using statistical software (StatPlus:Mac 2009, AnalystSoft Inc, Vancouver, BC, Canada). Comparisons of continuous variables were performed using the Wilcoxon sign-ranked test and comparison of categorical variables was performed using the Chi-squared test or Fisher Exact test. A $P$ value of $<0.05$ was considered as statistically significant.

\section{Results}

\section{Baseline demographics}

A total of 13 eyes from 11 patients were included in the study (Table 1). Two patients had bilateral CMV anterior segment inflammation with corneal endotheliitis presented in both

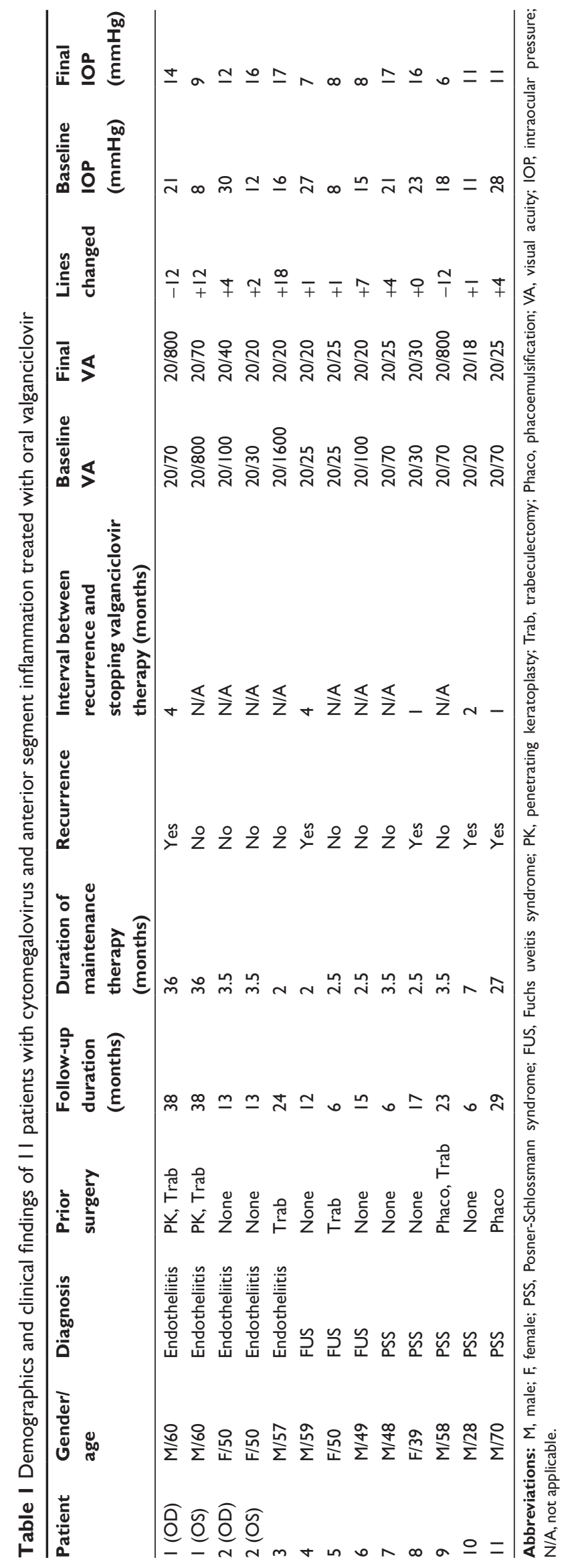


eyes at the same time. The mean age of the patients at the commencement of valganciclovir treatment was $51.6 \pm 10.8$ (range 28-70) years, and there were eight males and three females. All patients were of Chinese ethnicity. Diagnoses included Posner-Schlossmann syndrome in five (45.4\%) patients, Fuchs uveitis syndrome in three $(27.3 \%)$ patients, and corneal endotheliitis in three $(27.3 \%)$ patients.

All patients had aqueous aspiration performed prior to commencement of valganciclovir, and the PCR of the aqueous fluid was positive for CMV DNA and negative for herpes simplex virus and herpes zoster virus DNA. At time of starting oral valganciclovir, the mean \pm standard deviation $\log$ MAR visual acuity of the 13 eyes was $0.58 \pm 0.57$ (Snellen equivalent 20/76; range 0.0-1.8 logMAR units). The mean baseline IOP was $18.3 \pm 7.4$ (range 8-30) $\mathrm{mmHg}$ and the eyes were on a median three (range 0-4) types of antiglaucomatous eye drops prior to treatment with oral valganciclovir. Four (36.3\%) patients were also on oral acetazolamide for IOP control. Other topical medications used by the patients at presentation included $1 \%$ prednisolone acetate eye drops in seven patients, $0.5 \%$ loteprednol etabonate eye drops in three patients, and 1\% dexamethasone, neomycin, and polymyxin B combination eye drops in one patent. Six eyes had anterior chamber cells of $0.5+$ or more at initial presentation, and keratic precipitates were seen in nine $(69.2 \%)$ eyes. Five $(38.5 \%)$ eyes had iris atrophy at baseline. Six (46.2\%) eyes had previous surgery prior to the commencement of valganciclovir, including trabeculectomy (two eyes), keratoplasty (one eye), combined penetrating keratoplasty and trabeculectomy (one eye), phacoemulsification (one eye), and combined phacoemulsification and trabeculectomy (one eye).

\section{Changes in anterior segment inflammation}

The mean follow-up duration of the patients was 17.2 (range 6-38) months. All patients received a loading dose of oral valganciclovir $900 \mathrm{mg}$ twice daily for at least 2 weeks, with a median of 4 (range 2-9) weeks. This was followed by maintenance therapy with oral valganciclovir $450 \mathrm{mg}$ twice daily for 2-36 months. There was an absence of anterior segment inflammation in all patients within 4 weeks following commencement of oral valganciclovir. Repeat aqueous tap was performed in five $(45.5 \%)$ patients while they were taking valganciclovir, and the PCR for CMV was negative in all cases. Five (38.4\%) eyes of five $(45.5 \%)$ patients developed recurrence of anterior segment inflammation after stopping valganciclovir therapy. The median interval between stopping valganciclovir and recurrence of anterior segment inflammation was 2 (range 1-4) months. Two of these patients had repeat aqueous tap and both were found to be positive for CMV PCR. All patients were resumed on oral valganciclovir following recurrence of anterior segment inflammation. Three patients remained on valganciclovir at the last follow-up, two for prevention of IOP rise in Posner-Schlossmann syndrome and one for prevention of graft failure in corneal endotheliitis. Eight eyes underwent subsequent surgery during the follow-up period, including trabeculectomy in three eyes, combined phacoemulsification and trabeculectomy in two eyes, phacoemulsification and endothelial keratoplasty in one eye, and phacoemulsification in one eye.

\section{Changes in visual acuity and intraocular pressure}

At the last follow-up, the mean $\log$ MAR visual acuity was 0.37 (Snellen equivalent 20/47). There was a significant improvement in the level of visual acuity at the lastfollow-up compared with baseline (Wilcoxon sign-ranked test, $P=0.048)$. The mean visual improvement was 2.3 (range -12 to +18 ) lines. Two $(15.4 \%$ ) eyes lost three or more lines of vision, with one caused by failed corneal graft and the other due to bullous keratopathy.

For all 13 eyes in the study, the mean IOP at the final follow-up was $11.7 \pm 4.0$ (range 6-17) $\mathrm{mmHg}$. The reduction in IOP compared with baseline was statistically significant (Wilcoxon sign-ranked test, $P=0.021$ ). The median number of antiglaucomatous eye drops required per eye was 0 (range $0-3$ ) and the reduction from baseline was also statistically significant (Wilcoxon sign-ranked test, $P=0.004$ ). None of the patients were on oral acetazolamide at the last follow-up.

There was no significant change in the mean IOP for the eight eyes which did not undergo trabeculectomy during the follow-up period (Wilcoxon sign-ranked test, $P=0.29$ ). Nonetheless, the median number of antiglaucomatous eye drops for eyes which did not have trabeculectomy reduced significantly from three to one per eye following the use of oral valganciclovir (Wilcoxon sign-ranked test, $P=0.031$ ).

\section{Adverse events}

During the follow-up period, none of the patients developed any systemic adverse event while taking oral valganciclovir. Blood tests, including complete blood count and renal function test, were performed for all patients on oral valganciclovir as standard-of-care and they remained normal throughout the course of valganciclovir treatment. 


\section{Illustrative case}

A 70-year-old Chinese man (patient 11) was referred with increased IOP in his left eye and recurrent episodes of uveitis for 6 months, in a Posner-Schlossmann syndrome picture. He had a history of uneventful phacoemulsification, with intraocular lens implantation in both eyes performed two years previously. On initial presentation, his left eye visual acuity was $20 / 40$. The left eye IOP was $20 \mathrm{mmHg}$ controlled with latanoprost, dorzolamide and timolol eye drops together with oral acetazolamide. Slit-lamp examination of the left eye showed multiple fine pigmented keratic precipitates with $1+$ cells in the anterior chamber (Figure 1A). The posterior chamber intraocular lens was well centered in the capsular bag with no evidence of dislocation. In view of the clinical features of Posner-Schlossmann syndrome, aspiration of aqueous fluid was obtained for viral PCR and was positive for CMV. The oral acetazolamide was stopped and he was put on a course of oral valganciclovir $900 \mathrm{mg}$ twice daily for 3 weeks followed by valganciclovir $450 \mathrm{mg}$ twice daily for 2 months. Two weeks later, there was considerable reduction in keratic precipitates and anterior chamber cells, and left eye vision improved to 20/35, with IOP reduced to $11 \mathrm{mmHg}$. At one month after starting valganciclovir, the keratic precipitates completely resolved, with absence of cells in the anterior chamber (Figure 1B). The left eye visual acuity improved further to 20/25 with an IOP of $10 \mathrm{mmHg}$. The antiglaucomatous eye drops were reduced to timolol only. Repeat aqueous aspirate for CMV PCR was negative

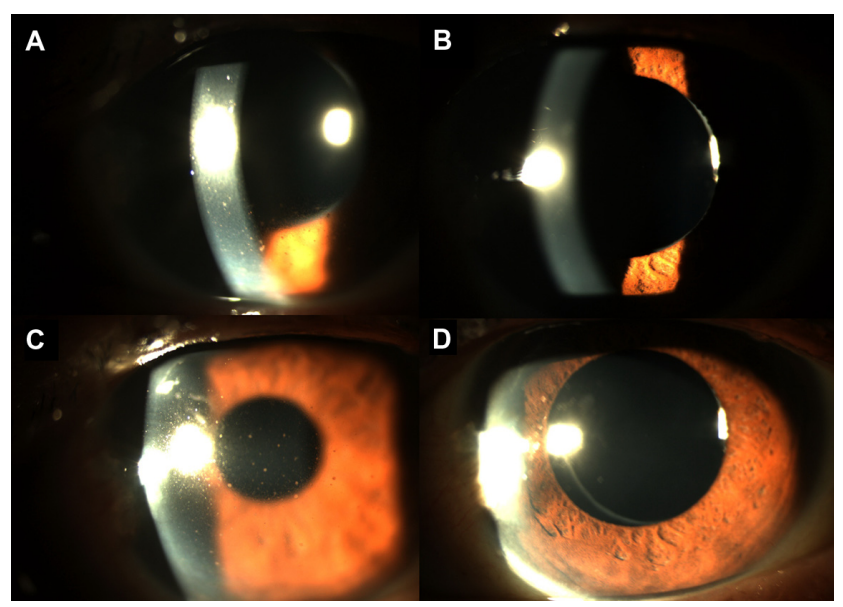

Figure I Slit-lamp photos of the left eye of a patient with anterior segment inflammation from cytomegalovirus. (A) Prior to commencement of valganciclovir, there were diffuse multiple pigmented keratic precipitates on the cornea. (B) Four weeks after valganciclovir therapy, the keratic precipitates completed resolved with absence of inflammation in the anterior chamber. (C) One month after stopping valganciclovir therapy, the was recurrence of keratic precipitates on the cornea with mild anterior chamber inflammation. (D) Two weeks after recommencing valganciclovir, the keratic precipitates again resolved with no inflammation in the anterior chamber. at 3 months after starting valganciclovir and the oral valganciclovir was then stopped.

One month after stopping valganciclovir, the patient complained of blurring of vision in the left eye. His left eye visual acuity reduced to 20/30 and the left eye IOP raised to $28 \mathrm{mmHg}$. Slit-lamp examination showed recurrence of left eye anterior uveitis with pigmented keratic precipitates and $1+$ cells in the anterior chamber (Figure 1C). Repeat aqueous aspiration was performed and the CMV PCR was positive. Oral valganciclovir was restarted at $450 \mathrm{mg}$ twice daily, and 2 weeks later the anterior segment inflammation resolved completely (Figure 1D). His vision improved back to $20 / 25$ and the left eye IOP reduced to $11 \mathrm{mmHg}$. The oral valganciclovir was maintained for 6 months with no recurrence of uveitis, and the IOP was controlled at around $10 \mathrm{mmHg}$ with timolol eye drops.

At 6 weeks after stopping valganciclovir for the second time, the patient again developed blurring of vision. The left eye visual acuity dropped to $20 / 50$ and the IOP was $32 \mathrm{mmHg}$. Slit-lamp examination showed left eye diffuse keratic precipitates with $1+$ cells in the anterior chamber. He was restarted on valganciclovir $450 \mathrm{mg}$ twice daily and the anterior segment inflammation again responded rapidly. Two weeks later, his left eye visual acuity improved to 20/30 and the IOP was $8 \mathrm{mmHg}$. The valganciclovir was maintained for over one year and there was no further recurrence of uveitis while the patient was on valganciclovir.

\section{Discussion}

Valganciclovir is a L-valyl ester prodrug of ganciclovir and provides excellent bioavailability following oral administration, with a plasma concentration similar to that of intravenous ganciclovir. ${ }^{14}$ It has been used to treat various ophthalmic disorders, including anterior uveitis, ${ }^{10-12} \mathrm{CMV}$ retinitis, ${ }^{14,15}$ acute retinal necrosis, ${ }^{16}$ immune recovery uveitis with macular edema, ${ }^{17}$ recurrent corneal allograft failure, ${ }^{18}$ and CMV corneal endotheliitis. ${ }^{19,20}$ Most of the studies published on the use of oral valganciclovir for CMV anterior segment inflammation have only reported short-term efficacy, and its longer-term efficacy is unclear.

In our current study, we evaluated the use of oral valganciclovir for treating anterior segment inflammation caused by CMV infection, and all patients had a minimum follow-up period of 6 months. Our results demonstrated that all patients had a good response, with complete resolution of anterior segment inflammation following the use of oral valganciclovir. The findings are consistent with previous reports that valganciclovir is an effective treatment for 
anterior uveitis and corneal endotheliitis due to CMV infection. ${ }^{4,5,10-12,20}$ Moreover, the long-term efficacy appears to be favorable because patients have significant visual gain after treatment, with a significant IOP reduction due to control of inflammation. The number of antiglaucomatous eye drops required by the patient also reduced significantly when compared with baseline in both eyes with or without trabeculectomy performed. The increase in IOP caused by CMV infection is likely to be due to viral trabeculitis and obstruction of trabecular meshwork by inflammatory debris. ${ }^{4}$ Therefore, the IOP can be controlled following control of anterior segment inflammation with antiviral therapy. The exact reason why some cases of CMV anterior segment inflammation develop an increase in IOP while others do not is unclear, but might be related to viral load or the extent of inflammation.

Several previous studies have demonstrated the efficacy of oral valganciclovir in treating anterior segment inflammation caused by CMV infection. Van Boxtel et al reported the outcomes for five patients with CMV anterior uveitis treated with oral valganciclovir for a mean duration of 8 (range 3-12) months. ${ }^{4}$ It was found that inflammation became quiescent within a few weeks following oral valganciclovir, and that the antiglaucomatous eye drops could be reduced shortly after treatment. However, recurrence was not uncommon and one patient had recurrence of uveitis despite having treatment for 12 months. Another patient also had recurrence when the dosage of valganciclovir was reduced. In another study by Chee et al, it was found that CMV infection was responsible for around $37.5 \%$ of patients with presumed Posner-Schlossmann syndrome and $31.3 \%$ of those with Fuchs heterochromic iridocyclitis. ${ }^{5}$ The authors also reported that all eyes responded well to the initial antiviral treatment, including oral valganciclovir or intravitreal ganciclovir, in terms of reducing inflammation and IOP control. However, $77.7 \%$ of cases relapsed within eight months after stopping antiviral treatment. ${ }^{5}$

As with previously published series, we also observed a high recurrence rate of anterior segment inflammation after stopping oral valganciclovir therapy because $38.5 \%$ of eyes developed recurrence during the follow-up period. The main reason for the high recurrence rate of CMV infection after stopping therapy is that all anti-CMV drugs, including ganciclovir and valganciclovir, are virustatic and not virucidal. ${ }^{21}$ In order to reduce the recurrence rate of CMV anterior segment inflammation after cessation of valganciclovir, other forms of ganciclovir treatment such as intravitreal or topical treatment have been suggested. ${ }^{10,12}$
Chee and Jap evaluated the use of systemic, topical, and intravitreal ganciclovir in 72 eyes from 70 patients with CMV anterior uveitis and found that topical ganciclovir gel had a moderate response rate but a lower recurrence rate compared with systemic or intravitreal ganciclovir. ${ }^{10}$ Huang et al evaluated the use of intravitreal ganciclovir injection as a loading dose with or without subsequent oral valganciclovir to treat $\mathrm{CMV}$ anterior uveitis. Two patients had only the initial intravitreal ganciclovir injection without oral valganciclovir and four patients had a mean of 2.3 months of oral valganciclovir after intravitreal treatment. The authors found that none of the patients had a recurrence of intraocular inflammation and all patients had significant improvement in visual acuity with significantly reduced IOP. Therefore, adding intravitreal ganciclovir might be a useful option in treating patients with CMV anterior uveitis. However, intravitreal ganciclovir frequently requires regular injections, and repeated intravitreal injections are associated with increased risks of intraocular infection, retinal break, and detachment. Because CMV anterior uveitis is less visionthreatening compared with CMV retinitis, we only used oral valganciclovir to treat patients with $\mathrm{CMV}$ anterior segment inflammation in order to avoid the potential risks associated with intravitreal ganciclovir injections.

At present, there is no consensus on the standard treatment duration when using valganciclovir for managing patients with CMV anterior segment inflammation. Based on our series and previous studies, ${ }^{8-10,20}$ significant clinical improvements can be observed within 2-4 weeks after starting antiviral therapy and, therefore, an initial treatment duration of at least 6 weeks appears to be a reasonable choice. In our series, three patients were still on valganciclovir at the last follow-up due to multiple recurrences after stopping valganciclovir. One patient with corneal endotheliitis who had penetrating keratoplasty will likely remain on long-term oral valganciclovir therapy in order to prevent graft failure caused by recurrence of corneal endotheliitis. Two patients in our series developed visual loss due to graft failure and bullous keratopathy associated with CMV corneal endotheliitis. This emphasizes the need for early control of CMV infection in these patients. Further long-term studies will be useful in determining the optimal treatment regimen and duration of maintenance valganciclovir therapy in patients with CMV anterior segment inflammation.

In summary, our study demonstrated that oral valganciclovir is useful in controlling anterior segment inflammation secondary to CMV infection. With the control of inflammation, the drug is effective in reducing IOP and 
the use of antiglaucomatous eye drops in these patients with CMV anterior segment inflammation. The main limitation of this study is its retrospective nature and the limited number of cases in the series, which prevented us from providing a definitive treatment recommendation for using oral valganciclovir for CMV anterior segment inflammation. Finding an alternative agent to replace oral valganciclovir might be useful in the long term due to the potential risk of systemic side effects associated with valganciclovir, such as neutropenia, anemia, and diarrhea. ${ }^{14,22}$ Nonetheless, none of the patients in our series developed any systemic adverse event associated with valganciclovir treatment. Another concern about oral valganciclovir therapy is the high cost of treatment because each $450 \mathrm{mg}$ tablet of valganciclovir costs around US\$30 and an initial 8 weeks of treatment will cost more than US\$4000. Therefore, further studies to evaluate the role of other alternative forms of ganciclovir in treating patients with CMV anterior uveitis as a more cost-effective monotherapy or combination therapy are warranted.

\section{Disclosure}

The authors report no conflicts of interest in this work.

\section{References}

1. Markomichelakis NN, Canakis C, Zafirakis P, Marakis T, Mallias I, Theodossiadis G. Cytomegalovirus as a cause of anterior uveitis with sectorial iris atrophy. Ophthalmology. 2002;109(5):879-882.

2. Bloch-Michel E, Dussaix E, Cerqueti P, Patarin P. Possible role of cytomegalovirus infection in the etiology of the Posner-Schlossmann syndrome. Int Ophthalmol. 1987;11(2):95-96.

3. Teoh SB, Thean L, Koay E. Cytomegalovirus in aetiology of Posner-Schlossman syndrome: evidence from quantitative polymerase chain reaction. Eye (Lond). 2005;19(12):1338-1340.

4. van Boxtek LA, van der Lelik A, van der Meer J, Los LI. Cytomegalovirus as a cause of anterior uveitis in immunecompetent patients. Ophthalmology. 2007;114(7):1358-1362.

5. Chee SP, Bacsal K, Jap A, Se-Thoe SY, Cheng CL, Tan BH. Clinical features of cytomegalovirus anterior uveitis in immunocompetent patients. Am J Ophthalmol. 2008;145(5):843-840.

6. Chee SP, Jap A. Presumed Fuchs heterochromic iridocyclitis and PosnerSchlossman syndrome: comparison of cytomegalovirus-positive and negative eyes. Am J Ophthalmol. 2008;146(6):883e1-889e1.
7. Van Gelder RN. Idiopathic no more: clues to the pathogenesis of Fuchs heterochromic iridocyclitis and glaucomatocyclitic crisis. Am J Ophthalmol. 2008;145(5):769-771.

8. Hwang YS, Shen CR, Chang SH, et al. The validity of clinical feature profiles for cytomegaloviral anterior segment infection. Graefes Arch Clin Exp Ophthalmol. 2011;249(1):103-110.

9. de Schryver I, Rozenberg F, Cassoux N, et al. Diagnosis and treatment of cytomegalovirus iridocyclitis without retinal necrosis. Br JOphthalmol. 2006;90(7):852-855.

10. Chee SP, Jap A. Cytomegalovirus anterior uveitis: outcome of treatment. Br J Ophthalmol. 2010;94(12):1648-1652.

11. Yamauchi Y, Suzuki J, Sakai J, Sakamoto S, Iwasaki T, Usui M. A case of hypertensive keratouveitis with endotheliitis associated with cytomegalovirus. Ocul Immunol Inflamm. 2007;15(5):399-401.

12. Hwang YS, Lin KK, Lee JS, et al. Intravitreal loading injection of ganciclovir with or without adjunctive oral valganciclovir for cytomegalovirus anterior uveitis. Graefes Arch Clin Exp Ophthalmol. 2010;248(2):263-269.

13. Martin DF, Sierra-Madero J, Walmsley S, et al. A controlled trial of valganciclovir as induction therapy for cytomegalovirus retinitis. N Engl J Med. 2002;34615(15):1119-1126.

14. Patil AJ, Sharma A, Kenney MC, Kuppermann DB. Valganciclovir in the treatment of cytomegalovirus retinitis in HIV-infected patients. Clin Ophthalmol. 2010;4:111-119.

15. Stewart MW. Optimal management of cytomegalovirus retinitis in patients with AIDS. Clin Ophthalmol. 2010;4:285-299.

16. Savant V, Saeed T, Denniston A, Murray PI. Oral valganciclovir treatment of varicella zoster virus acute retinal necrosis. Eye (Lond). 2004;18(5):544-545.

17. Kosobucki BR, Goldberg DE, Bessho K, et al. Valganciclovir for immune recovery uveitis complicated by macular edema. Am J Ophthalmol. 2004;137(7):636-638.

18. Lusthaus JA, Kim P, Steller AK, et al. Successful corneal autograft after clearance of anterior chamber cytomegalovirus with oral valganciclovir in a patient with multiple failed cornea allografts. Cornea. 2011;30(9):1054-1057.

19. Park UC, Kim SJ, Yu HG. Cytomegalovirus endotheliitis after fluocinolone acetonide (Retisert) implant in a patient with Behcet uveitis. Ocular Immunol Inflamm. 2011;19(4):282-283.

20. Chee SP, Jap A. Treatment outcome and risk factors for visual loss in cytomegalovirus endotheliitis. Graefes Arch Clin Exp Ophthalmol. 2012;250(3):383-389.

21. Lalonde RG, Boivin G, Deschenes J, et al. Canadian consensus guidelines for the management of cytomegalovirus disease in HIV/AIDS. Can J Infect Dis Med Microbiol. 2004;15(6):327-335.

22. Lalezari J, Lindley J, Walmsley S, et al. A safety study of oral valganciclovir maintenance treatment of cytomegalovirus retinitis. J Acquir Immune Defic Syndr. 2002;30(4):392-400.
Clinical Ophthalmology

\section{Publish your work in this journal}

Clinical Ophthalmology is an international, peer-reviewed journal covering all subspecialties within ophthalmology. Key topics include: Optometry; Visual science; Pharmacology and drug therapy in eye diseases; Basic Sciences; Primary and Secondary eye care; Patient Safety and Quality of Care Improvements. This journal is indexed on Submit your manuscript here: http://www.dovepress.com/clinical-ophthalmology-journal

\section{Dovepress}

PubMed Central and CAS, and is the official journal of The Society of Clinical Ophthalmology (SCO). The manuscript management system is completely online and includes a very quick and fair peer-review system, which is all easy to use. Visit http://www.dovepress.com/ testimonials.php to read real quotes from published authors. 